\title{
Using a non-invasive multi-sensor device to evaluate left atrial pressure: an estimated filling pressure derived from ballistocardiography
}

\author{
Li Zhang ${ }^{1,2}$, Peiwei $\mathrm{Cai}^{3}$, Yinlong Deng ${ }^{1}$, Jiumin $\mathrm{Lin}^{4}$, Muli Wu ${ }^{1}$, Zhongbo Xiao ${ }^{1}$, Zhengpei Chu ${ }^{5}$, \\ Qingfeng Shi ${ }^{5}$, Fei Ye ${ }^{5}$, Junhao $\mathrm{Hu}^{5}$, Chao Yang ${ }^{5}$, Pengyang $\mathrm{Li}^{6}$, Shaochun Zhuang ${ }^{7}$, Bin Wang ${ }^{1,2}$ \\ ${ }^{1}$ Department of Cardiology, the First Affiliated Hospital of Shantou University Medical College, Shantou, China; ${ }^{2}$ Clinical Research Center, the First \\ Affiliated Hospital of Shantou University Medical College, Shantou, China; ${ }^{3}$ Ultrasound Division, the First Affiliated Hospital of Shantou University \\ Medical College, Shantou, China; ${ }^{4}$ Department of Hepatology and Infectious Diseases, the Second Affiliated Hospital of Shantou University Medical \\ College, Shantou, China; ${ }^{5}$ DARMA Lab, Shenzhen, China; ${ }^{6}$ Department of Medicine, Saint Vincent Hospital, Worcester, MA, USA; ${ }^{7}$ Cardiostory \\ Inc., Lewes, DE, USA \\ Contributions: (I) Conception and design: B Wang, L Zhang; (II) Administrative support: B Wang, P Cai; (III) Provision of study materials or patients: \\ L Zhang, P Cai, Y Deng; (IV) Collection and assembly of data: Y Deng, L Zhang, M Wu, Z Xiao; (V) Data analysis and interpretation: Y Deng, J \\ Lin; (VI) Manuscript writing: All authors; (VII) Final approval of manuscript: All authors. \\ Correspondence to: Bin Wang, MD, PhD. Department of Cardiology, the First Affiliated Hospital of Shantou University Medical College, 57 \\ Changping Road, Shantou 515041, China; Clinical Research Center, the First Affiliated Hospital of Shantou University Medical College, 57 \\ Changping Road, Shantou 515041, China. Email: wangbin_pku@126.com; Shaochun Zhuang. Cardiostory Inc., 16192 Coastal Highway, Lewes, DE \\ 19958, USA. Email: shawn.z@cardiostory.com.
}

Background: Heart failure is a global health problem, and elevated left atrial pressure (LAP) is a precursor to identifying decompensated heart failure. At present, out-of-hospital monitoring of patients with heart failure is mostly based on the patient's symptoms and signs, and the use of non-invasive technology is scarce. In this study, a non-invasive ballistocardiography (BCG) device was used to collect thoracic vibration signals generated by heartbeat. We collected these signals from more than 1,000 adults, including those with different heart diseases, and used a sensor system and a composite index related to LAP recognition named the LAP-index, to analyze them. This study aimed to verify the reliability and accuracy of the LAP-index in identifying elevated LAP within heart failure patients.

Methods: We prospectively included 158 patients with various extent of diastolic function, some of whom had various underlying diseases, and collected BCG and echocardiographic data using a cross-section methodology. The BCG signal was recorded from multiple optical fiber vibration sensors placed on the back of each patient. We adopted the 2016 ASE/EACVI echocardiography guideline as the standard for determining LAP level from echocardiography parameters. To evaluate the diagnostic efficacy of the LAP-index, we drew a receiver operating characteristic (ROC) curve and calculated the area under the ROC curve (AUC).

Results: The LAP-index of the 158 patients ranged from 6 to 32. Of them, 39 were diagnosed as high LAP by echocardiography, and 119 cases had normal or slightly elevated LAP. Comparison of the LAP-index results and echocardiographic results revealed the ROC c-statistic of the LAP-index for identifying high LAP was 0.86 (95\% CI: 0.79-0.93; P<0.0001). When the LAP-index was at the best cut-off value of 15.5 , the positive agreement rate between it and echocardiography LAP was 0.85 , the negative agreement rate was 0.80 , and the overall agreement rate was 0.81 .

Conclusions: The sensor system and the LAP-index, a composite index derived from BCG, have high reliability and accuracy in identifying elevated LAP, which provides a novel possibility for the non-invasive detection of hemodynamic congestion in heart failure patients.

Keywords: Ballistocardiography (BCG); atrial pressure; heart failure 
Submitted Aug 31, 2021. Accepted for publication Oct 20, 2021.

doi: 10.21037/atm-21-5161

View this article at: https://dx.doi.org/10.21037/atm-21-5161

\section{Introduction}

Heart failure is a complex set of clinical syndromes and the final stage of various cardiovascular diseases. Although the past few decades have seen significant progress in drugs and other equipment to treat the condition, the hospitalization rate and rehospitalization rate remain high, and the 5-year mortality rate is as high as $50 \%$ (1). The hospitalization rate is a powerful independent predictor of mortality in heart failure, as it often indicates patients have entered a decompensation period when it is difficult to restore heart structure and function, even with treatment $(2,3)$. Therefore, it is crucial to reduce the hospitalization rate by detecting various physiological parameters and assessing the progress of heart failure at an early stage and establish intervention measures in a timely manner (3).

Traditional methods for assessing the disease progression of patients with heart failure include monitoring symptoms and signs and recording the daily weight $(4,5)$, and monitoring natriuretic peptide levels (6), noninvasive bioimpedance (7), and implantable bioimpedance (8). While the advantage of monitoring body weight to assess acute decompensation in heart failure is easy to quantify, the sensitivity is low $(9,10)$. Compared with transthoracic impedance (10), albeit a with a higher false-positive rate in predicting acute heart failure events (10-12). The monitoring and management of these traditional indicators have a low sensitivity and high false-positive rate, and relevant clinical trials have not confirmed their use results in a reduction in hospitalization rates (13).

At present, the most well-recognized management indicator of heart failure is the monitoring of ventricular filling pressure. Left ventricular filling pressure (LVFP) can be indicated by pulmonary capillary wedge pressure (PCWP), left atrial pressure (LAP), or left ventricular end-diastolic pressure (LVEDP) (14), and elevated LAP is a precursor to decompensated heart failure. In the past, filling pressure has been assessed by carotid pulsation, edema, dyspnea, rales, the presence of a third heart sound, pulse pressure, and other symptoms and signs (15). While the use of right heart catheter to measure PCWP and left heart catheters to measure LVFP are the gold standards, invasive real-time measurement methods are limited to use by professional medical hospital staff. The CHAMPION clinical trial in 2011 showed that the use of pulmonary arterial pressure (PAP) detected by implantable devices in the management of heart failure reduced its hospitalization rate by $37 \%$ (10). By maintaining PAP in the target range, patients can ultimately reduce the acute decompensation events of heart failure (16). In addition to monitoring filling pressure by implanted pressure sensors, the third sound (S3) and a composite index combined with $\mathrm{S} 3$ to monitor the LVFP of patients with heart failure has been used. In 2017, Thakur $e t$ al. found that found that variations of heart sound were highly correlated with LAP on animal models (17). Researchers used multiple sensors on implanted devices to build an algorithm involving the core parameter of heart sounds and several other parameters related to heart failure, including heart rate, respiration, transthoracic impedance, and range of motion. The results showed that its sensitivity for warning of impending acute decompensation of heart failure was $70 \%$, the number of false alarms per year was 1.4 times per person, and its ability to warn of the acute worsening of heart failure could be determined on average 34 days in advance (18). However, implantation methods are invasive, costly, and require highly trained professional medical staff (16). Therefore, there is an urgent need for alternative approaches, especially the use of non-invasive devices.

Ballistocardiography (BCG) is a non-invasive technique for monitoring cardiovascular function. By using a novel fiber-optic multi-sensor device to collect BCG signals, this technology can effectively record the heartbeat and the thoracic vibrations it produces during different cardiac phases and heart function can be evaluated by analyzing the amplitude and frequency of the vibrations (19). There is no report of a non-invasive product or technology for evaluating filling pressure based on BCG signals previously. Prior to this study, another study has been reported by our team in July 2021 (20), demonstrating the amplitude of the S3 in BCG signal collected by fiber optic sensors can be used to identify patient with elevated filling pressure determined by echocardiography parameters. In that observational study, a single signal feature of S3 amplitude is studied and evaluated, demonstrating feasibility of using the $\mathrm{S} 3$ signal feature derived from BCG signal to identify elevated filling pressure. After that feasibility study, more BCG data were collected 


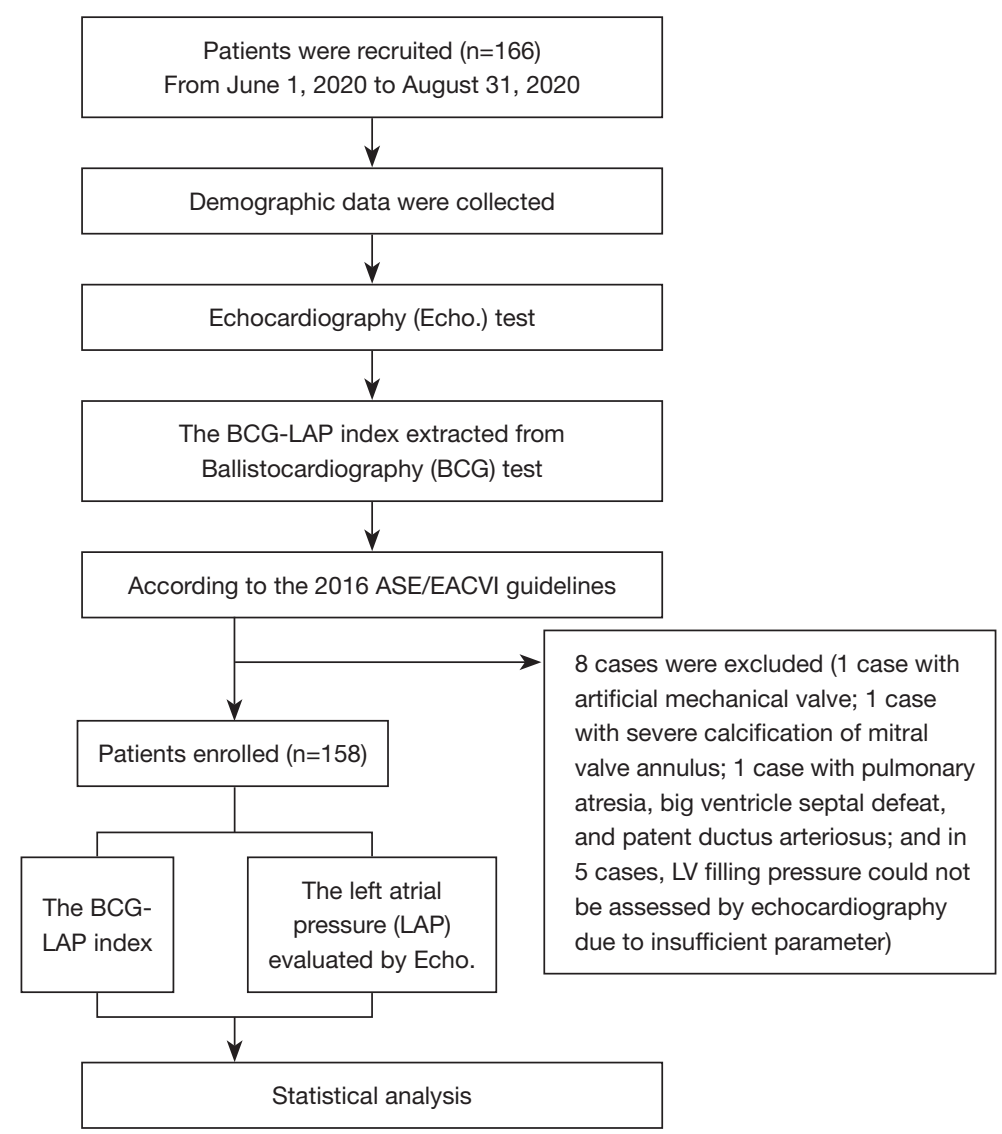

Figure 1 Study flowchart. ASE, the American Society of Echocardiography; LAP, left atrial pressure; The BCG-LAP index, physiological parameters related to left atrial pressure by ballistocardiography; ECHO, echocardiographic.

from over 1,000 patients with corresponding comprehensive echocardiography reports. This generated a data base for us to development an algorithm, which can automatically identify multiple feature parameters from BCG signal and calculated an estimated filling pressure, named the LAPindex. In this study, the sensors system and the algorithm is studied to verify the reliability and accuracy of the LAP-index output in identifying high LAP status in patients with various cardiac diastolic function compared with echocardiography. We present the following article in accordance with the STARD reporting checklist (available at https://dx.doi. org/10.21037/atm-21-5161).

\section{Methods}

\section{Study population}

All procedures performed in this study involving human participants were in accordance with the Declaration of
Helsinki (as revised in 2013). The research project was approved by the Ethical Review Committee of the First Affiliated Hospital of Shantou University Medical College (No. 2019071). Before participating in the clinical study, all patients signed informed consent. This was a prospective, single-center diagnostic study conducted to verify the accuracy and reliability of the LAP-index derived from the BCG signal in identifying the LAP. From June 1, 2020, to August 31, 2020, we enrolled 166 subjects in the echocardiography room of the First Affiliated Hospital of Shantou University Medical College and collected the BCG and echocardiographic parameters from the patients by cross-section. A flow chart of this research is shown in Figure 1.

Inclusion criteria: age $\geq 18$ years; inpatients in cardiology, including patients with heart failure and other heart diseases; other non-cardiac inpatients, including corresponding internal and surgical specialist diseases and/or patients with combined heart disease; and some outpatients. In 


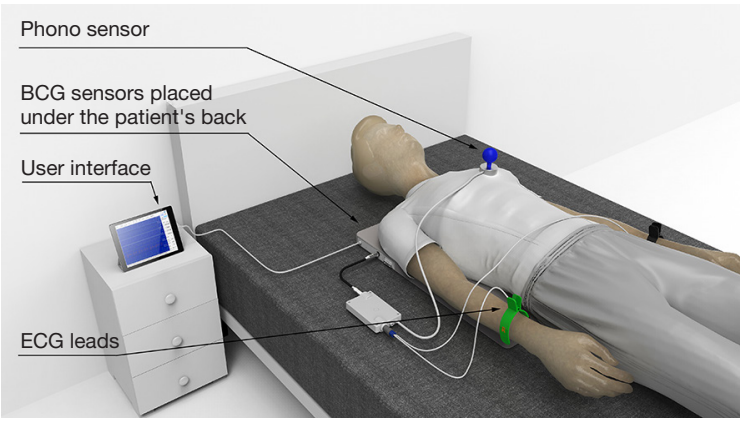

Figure 2 Patients lay flat on a BCG sensor module containing fiber optics, with a traditional heart sound sensor attached to their chest. The ECG leads contact the patient's upper limbs by clips. BCG, ballistocardiography; ECG, electrocardiogram.

this study, the definition of heart failure is based on 2021 Universal Definition and Classification of Heart Failure (21): (I) symptoms and or signs caused by a structural and/or functional cardiac abnormality (as determined by an EF of $<50 \%$, abnormal cardiac chamber enlargement, E/e' of $>15$, moderate/severe ventricular hypertrophy or moderate/severe valvular obstructive or regurgitant lesion); (II) and corroborated by at least one of the following: (I) elevated natriuretic peptide levels; (II) objective evidence of cardiogenic pulmonary or systemic congestion by diagnostic modalities, such as imaging (e.g., by chest radiograph or elevated filling pressures by echocardiography) or hemodynamic measurement (e.g., right heart catheterization, pulmonary artery catheter) at rest or with provocation (e.g., exercise).

Exclusion criteria: patients with severe mitral valve annulus calcification; patients with prosthetic valves or annulus; patients with congenital heart disease; and patients whose LAP could not be graded because some parameters of echocardiography could not be obtained.

\section{$B C G$}

\section{Introduction to equipment principle}

During the BCG examination, the patient was supine, with the BCG sensors containing optical fiber placed under their back. Chest vibration caused by the movement of the patient's heart and the blood flow in the large blood vessels causes the optical fiber to produce slight bending and deformation, and by analyzing variations in the light signal caused by this deformation, we obtained the BCG signal trace. Since the optical signal is not interfered with by external electromagnetic signals, it can be amplified as extremely subtle variations can be detected. Figure 2 shows the detection scenario.

The BCG signal acquisition system was designed and developed by the Shenzhen DARMA Technology Co. Ltd, and the optical fiber sensor components manufactured by the Shenzhen Fiber Medical Technology Co. Ltd. Part of the algorithm was jointly developed by the Shenzhen DARMA Technology Co., Ltd. and Cardiostory Inc., Delaware, USA.

\section{Stability and repeatability of BCG signal}

This research was based on sensor technology which can obtain highly repeatable BCG signals. Figure $3 A$ shows a subject with eight consecutive heartbeat signals, in which the electrocardiogram (ECG), phonocardiography (PCG), and BCG were collected simultaneously, and reveals the signals between multiple heartbeats had a high degree of similarity. Figure $3 B$ illustrates the trajectory diagram formed by superimposing the BCG signals of multiple cardiac cycles.

\section{Core technology}

The core technology of this research was an optical fiber sensor based comprehensive algorithm, which uses the third heart sound as the major parameter. Based on non-invasive hemodynamic information, the comprehensive algorithm LAP-index was developed and refined based on the BCG signals of over 1,000 patients with different heart diseases in multi-centers. The LAP-index ranged from 5 to 32, and the larger the value, the larger the estimated LAP.

\section{Stability of the BCG signal acquisition system and LAP calculation algorithm}

To avoid selection bias, we collected BCG signals from 15 patients with heart disease, including different underlying diseases, on the same day in the cardiology inpatient ward in sequence of bed numbers. After continuously recording the BCG signal from the patients for 10 minutes in a quiet and supine position, we divided the data into different time lengths, including the first 1, 2, 3, 5 and 8 minutes to analyze the stability.

\section{Repeatability of BCG signal acquisition system and LAP calculation algorithm}

After verifying the stability of the acquisition time, we then verified the repeatability of the BCG signal acquisition system and algorithm. For four consecutive days, we 


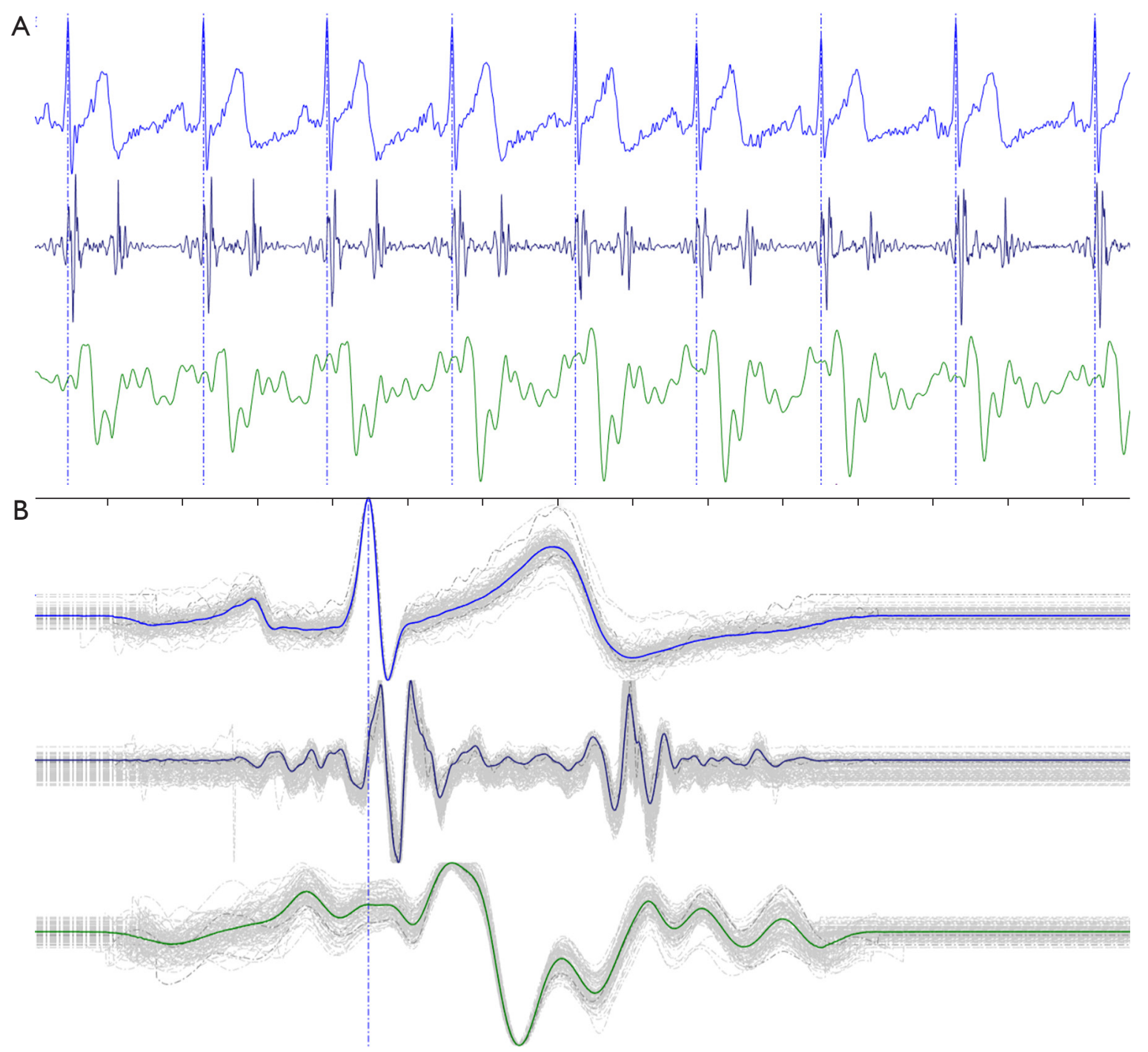

Figure 3 The stability and the repeatability of BCG signal. (A) The signal shape of BCG is shown in the three traces from top to bottom: ECG, PCG, and BCG; (B) the BCG signal is a trace formed by the superposition of 100 beats. The solid line represents the average trace after superposition. BCG, ballistocardiography; ECG, electrocardiogram; PCG, phonocardiography.

collected BCG signals from 30 patients with heart disease, including different underlying diseases, in the cardiology inpatient ward in sequence of bed numbers. The signals of each patient were collected for 1 minute in a quiet and supine position, five times in a row.

\section{Accuracy verification of the system and algorithm}

We recruited 158 patients as a test set to independently validate the performance of the sensor system and the LAPindex. BCG signals were acquired with 5 minutes right after an echocardiographic examination, and we compared the collected LAP-index results with the LAP evaluation results from the echocardiographic parameters. The team members operating BCG measurement and recording the LAP-index result are blinded to echocardiography parameters and result.

\section{Echocardiography}

We used a Philips5500 and Doppler tissue imaging (DTI) device equipped with a multi-frequency transducer to collect ultrasound images of patients in the echocardiography clinic. A standard view was used to perform a comprehensive examination with all Doppler echocardiogram and DTI records were obtained during normal breathing. The pulse- 
Doppler sampling volume was placed at the apex of the mitral valve and recorded 5-10 cardiac cycles from the apical window. The DTI program was set to pulse wave Doppler mode and the filter was set to exclude high-frequency signals, with the Nyquist limit adjusted to the speed range of -15 to $20 \mathrm{~cm} / \mathrm{s}$. To obtain a clear tissue signal and minimum background noise, the gain was minimized, and a sample with a volume of $5 \mathrm{~mm}$ was placed on the inner side (or interval) of the mitral annulus on the four-chamber heart section under the xiphoid process.

Echocardiography image acquisition and analysis were performed by a technician, using an offline analysis station (MedEx) equipped with Doppler software. Personnel involved in the collection of ultrasound data and in the collection of BCG data were mutually blinded to the process. The end-diastolic and end-systolic volumes were calculated using the two-plane method and these volumes were then used to calculate the left ventricular ejection fraction (LVEF). A four-chamber view of the apical heart was used to measure the volume of the left atrium, and the tricuspid regurgitation velocity (VTR) to estimate the pulmonary artery systolic pressure. The mitral inflow peak early diastolic velocity (E) and mitral inflow peak late diastolic velocity (A) were recorded, then using DTI, the early diastolic velocity of the mitral annulus (e'-septal) and late diastolic velocity of the mitral annulus (a') in the mitral annulus interval, which was and measured three to five cardiac cycles to obtain an average value. In this study, the parameter e' is the septal e'.

We adopted the 2016 ASE/EACVI guidelines as the basis for the assessment and classification of LAP (14). If the mitral valve blood flow spectrum showed that the E/A ratio was $\leq 0.8$, and the peak E wave velocity was $\leq 50 \mathrm{~cm} / \mathrm{s}$, it indicated that the average LAP was normal or low, and the corresponding diastolic dysfunction was graded I. An E/A ratio $>2$ indicated the average LAP was significant elevated, and the abnormal diastolic function was graded as III. For patients with a transmitral blood flow E/A ratio $\leq 0.8$ and $\mathrm{E}$ wave peak velocity $>50 \mathrm{~cm} / \mathrm{s}$, or the E/A ratio $>0.8$ but $<2$, other indicators were consulted to further evaluate diastolic function. The expert group recommends the following indicators and their cut-off values. (I) Peak velocity of tricuspid regurgitation (VTR) $>2.8 \mathrm{~m} / \mathrm{s}$; (II) mean $\mathrm{E} / \mathrm{e}^{\prime}$ ratio $>14$; (III) maximum left atrium volume index (LAMVI) $>34 \mathrm{~mL} / \mathrm{m}^{2}$. If all three indicators can be measured, and only one is abnormal, it indicates that the LAP is normal, and the diastolic dysfunction is grade I. If there are two abnormalities in the three, or all three indicators are abnormal, an increase in LAP is indicated, and the diastolic dysfunction is grade II. For patients in whom only one index is measured, and in those who have two indexes measured but the results of the two indexes are inconsistent, the LAP should not be reported, nor should the diastolic dysfunction be classified. In this study, e' is taken from the septum. According to the 2016 ASE/EACVI guidelines, when only septum e' is available or effective, the ventricular septum $\mathrm{E} / \mathrm{e}^{\prime} \geq 15$ is considered abnormal clinically.

Based on the LAP-index results, patients were divided into three tertiles (E/e' $\left.\leq 8 ; 8<\mathrm{E} / \mathrm{e}^{\prime}<15 ; \mathrm{E} / \mathrm{e}^{\prime} \geq 15\right)$ for comparison between groups.

\section{Statistical analysis}

This study is a preliminary study on the recognition of high LAP by BCG equipment; the specific sample size cannot be calculated by statistics. All statistical analysis was performed by IBM SPSS 22.0. The statistical graph was drawn by GraphPad Prism 8.3. Continuous variables are expressed as mean \pm standard deviation and categorical variables and constituent ratios as percentage $\mathrm{N}$ (\%). Before comparison, each group of continuity variables was tested for normality and homogeneity of variance, and when $\mathrm{n} \geq 50$, the Kolmogorov-Smirnov (K-S) method was adopted for the normality test, and when $\mathrm{n}<50$, the Shapiro-Wilk (S-W) method was adopted. One-way ANOVA analysis was adopted to compare the variables of the three groups, and the LSD test in the post hoc analysis was adopted to compare the differences between the groups. To explore the diagnostic efficacy of the LAP-index, the receiver operating characteristic (ROC) curve was used, and the area under the ROC curve (AUC) was calculated. The cut-off value of the LAP-index was determined by the maximum value of the Youden index, and a paired four-grid table was adopted to compare the consistency of the LAP-index with the comprehensive interpretation method of echocardiography. When $\mathrm{P}$ value $<0.05$, the difference was considered statistically significant.

\section{Results}

\section{Stability of the BCG signal acquisition system and LAP calculation algorithm}

The LAP-index of different time lengths from 15 patients is listed in Table 1. Statistics show that the average intragroup correlation coefficient (ICC) was 0.98 (95\% CI: 0.95, 
Table 1 Stability of the BCG signal acquisition system and LAP calculation algorithm

\begin{tabular}{|c|c|c|c|c|c|}
\hline Items & 1 minute & 2 minutes & 3 minutes & 5 minutes & 8 minutes \\
\hline Subject 2 & 17 & 17 & 18 & 18 & 18 \\
\hline Subject 3 & 17 & 20 & 20 & 17 & 17 \\
\hline Subject 4 & 15 & 14 & 14 & 14 & 14 \\
\hline Subject 6 & 11 & 10 & 11 & 12 & 12 \\
\hline Subject 7 & 12 & 12 & 12 & 20 & 17 \\
\hline Subject 8 & 13 & 13 & 13 & 13 & 13 \\
\hline Subject 9 & 13 & 14 & 13 & 14 & 14 \\
\hline Subject 12 & 16 & 16 & 16 & 16 & 16 \\
\hline Subject 13 & 14 & 15 & 15 & 13 & 13 \\
\hline Subject 14 & 12 & 12 & 12 & 12 & 12 \\
\hline Subject 15 & 31 & 30 & 30 & 30 & 22 \\
\hline Mean \pm SD & $15.20 \pm 5.13$ & $15.33 \pm 5.10$ & $15.40 \pm 5.08$ & $15.67 \pm 5.05$ & $14.93 \pm 3.45$ \\
\hline
\end{tabular}

BCG, ballistocardiography; LAP, left atrial pressure.

Table 2 Repeatability test results of LAP-index

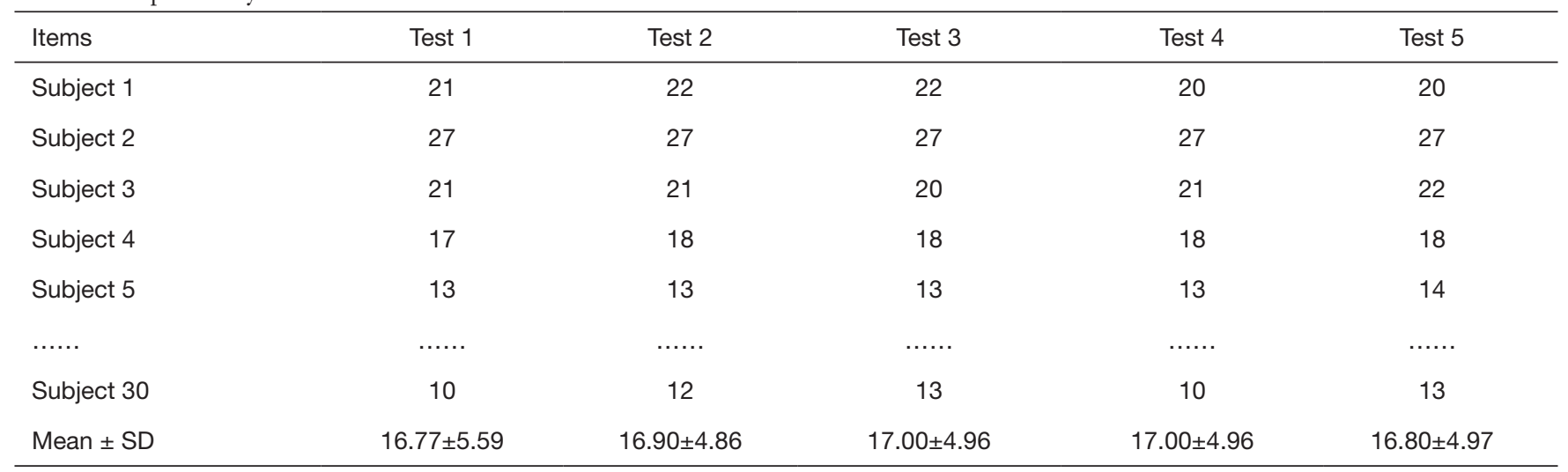

LAP, left atrial pressure.

0.99), $\mathrm{P}<0.0001$, and the reliability analysis revealed that the Cronbach's alpha coefficient was 0.98 .

\section{Repeatability of the BCG signal acquisition system and LAP calculation algorithm}

The 1-minute LAP-index obtained from 30 patients is shown in Table 2. Statistics showed the average ICC is 0.97
(95\% CI: $0.95,0.98), \mathrm{P}<0.0001$, and the reliability analysis revealed the Cronbach's alpha coefficient was 0.97 .

\section{Accuracy of the BCG signal acquisition system and LAP calculation algorithm}

Of the 166 recruited subjects, eight were excluded (one case with an artificial mechanical valve, one with 
Table 3 Demographic characteristics and clinical data

\begin{tabular}{|c|c|}
\hline Variable & $N=158$ \\
\hline Age (yr) & $59.6 \pm 15.0$ \\
\hline Male, $\mathrm{n}[\%]$ & 85 [53] \\
\hline BMI $\left(\mathrm{kg} / \mathrm{m}^{2}\right)$ & $23.0 \pm 13.0$ \\
\hline $\mathrm{SBP}(\mathrm{mmHg})$ & $130 \pm 19$ \\
\hline $\mathrm{DBP}(\mathrm{mmHg})$ & $77.8 \pm 12.2$ \\
\hline Heart rate (beats/min) & $76.5 \pm 9.7$ \\
\hline \multicolumn{2}{|l|}{ Clinical characteristics, n [\%] } \\
\hline Coronary artery disease & $31[19]$ \\
\hline Hypertension & $66[41]$ \\
\hline Hyperlipidemia & $12[7]$ \\
\hline Diabetes mellitus & 35 [22] \\
\hline Chronic kidney disease & 5 [3] \\
\hline \multicolumn{2}{|c|}{ Baseline medical therapy for CHF, n [\%] } \\
\hline Beta-blockers & 36 [22] \\
\hline ACE-I or ARB & $41[25]$ \\
\hline ARB/neprilysin inhibitor & 9 [5] \\
\hline Aldosterone receptor blocker & $21[13]$ \\
\hline Diuretics & $33[20]$ \\
\hline Digoxin & 6 [3] \\
\hline Other vasodilators & 9 [5] \\
\hline HF, n [\%] & 59 [37] \\
\hline NT-proBNP, n [\%] & 46 [29] \\
\hline NT-proBNP (pg/mL) & $2,046 \pm 4,767$ \\
\hline
\end{tabular}

Data presented are mean \pm SD or number of patients. BMI, body mass index; SBP, systolic blood pressure; DBP, diastolic blood pressure; ACE-I, angiotensin-converting enzyme inhibition; $A R B$, angiotension receptor blocker; $\mathrm{CHF}$, congestive heart failure; $\mathrm{HF}$, heart failure; NYHA, New York Heart Association; NT-proBNP, NT-proB-type natriuretic peptide.

severe calcification of the mitral valve annulus, one with pulmonary atresia, big ventricle septal defeat, and patent ductus arteriosus, and five cases, LV filling pressure could not be assessed by echocardiography due to insufficient parameter). Finally, 158 patients were evaluated with BCG and echocardiography, and Table 3 shows their demographic data, clinical history, and drug treatment history. Table 4 shows the echocardiographic and BCG characteristics of the patients, in whom the LAP-index ranged from
Table 4 Echocardiographic and ballistocardiography characteristics

\begin{tabular}{|c|c|}
\hline Variable & $N=158$ \\
\hline LVEF <50\% & $26(16 \%)$ \\
\hline e' septal available & $153(96.8 \%)$ \\
\hline e' septal $<7 \mathrm{~cm} / \mathrm{s}$ & 78 (49\%) \\
\hline E/e' available & 153 (96.8\%) \\
\hline E/e' septal <8 & $30(18.9 \%)$ \\
\hline $8 \leq$ E/e' septal $<15$ & 90 (56.9\%) \\
\hline E/e' septal $\geq 15$ & $40(25.3 \%)$ \\
\hline VTR available & 90 (56.9\%) \\
\hline VTR $\geq 280 \mathrm{~cm} / \mathrm{s}$ & $28(17.7 \%)$ \\
\hline LAMVI available & $153(96.8 \%)$ \\
\hline LAMVI >34 mL/m ${ }^{2}$ & $86(54.4 \%)$ \\
\hline $\begin{array}{l}\text { No. of patient with elevated filling } \\
\text { pressure by echocardiography }\end{array}$ & $\begin{array}{l}39 \text { (24.6\%), (17 with LVEF } \\
<50 \% ; 22 \text { with LVEF } \geq 50 \%)\end{array}$ \\
\hline $\begin{array}{l}\text { No. of patient with non- } \\
\text { elevated filling pressure by } \\
\text { echocardiography }\end{array}$ & $\begin{array}{c}119 \text { (75.4\%), (7 with } E F<50 ; \\
112 \text { with } E F \geq 50)\end{array}$ \\
\hline $\begin{array}{l}\text { Single cross mitral inflow peak } \\
\text { (suspected to be AF) }\end{array}$ & $21(13.2 \%)$ \\
\hline \multicolumn{2}{|c|}{$\begin{array}{l}\text { LVEF, left ventricular ejection fraction; E, mitral inflow peak early } \\
\text { diastolic velocity; e', early diastolic velocity of mitral annulus; E/e' } \\
\text { ratio, mitral inflow peak early diastolic velocity divided by early } \\
\text { diastolic velocity of mitral annulus; VTR, tricuspid regurgitation } \\
\text { velocity; LAMVI, left atrial maximal volume index; AF, atrial } \\
\text { fibrillation. }\end{array}$} \\
\hline
\end{tabular}

6 to 32. Based on the LAP-index results, patients were then divided into three groups (E/e' $\leq 8,8<\mathrm{E} / \mathrm{e}^{\prime}<15$, and E/e' $\geq 15$ ) (Figure 4). Comparisons were made for the three groups and showed that compared with the E/e' $\leq 8$ group $(13.10 \pm 3.74)$ and the $8<\mathrm{E} / \mathrm{e}^{\prime}<15$ group $(13.13 \pm 4.06)$, the E/e' $\geq 15$ group $(18.73 \pm 6.37)$ was significantly higher than the other two groups $(\mathrm{P}<0.0001)$. There was no significant statistical difference between the E/e' $\leq 8$ group and the $8<$ E/e' $<15$ group $(\mathrm{P}=0.9992)$. According to the 2016 ASE/ EACVI guidelines (14), among the 158 patients, 39 were diagnosed as high LAP, in whom the LAP-index of $29 \geq 18$, and in 10 cases $<18$. Among the 10 patients with an LAPindex $<18,3$ were 17,1 was 16,1 was 15 , and the other 5 were $<15$. Comparison of the LAP-index results and echocardiographic results revealed that the ROC c-statistic of the LAP-index for identifying high LAP was 0.86 (95\% CI: $0.79-0.93 ; \mathrm{P}<0.0001)$ (Figure 5). When the cut-off 


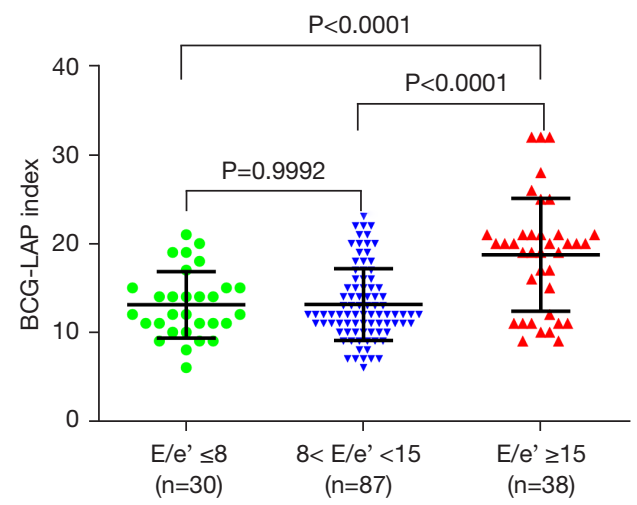

Figure 4 Comparison of BCG-LAP index among the three study groups. BCG-LAP index, parameters related to LAP derived by ballistocardiography; E/e', mitral inflow peak early diastolic velocity divided by early diastolic velocity of mitral annulus; BCG, ballistocardiography; LAP, left atrial pressure.

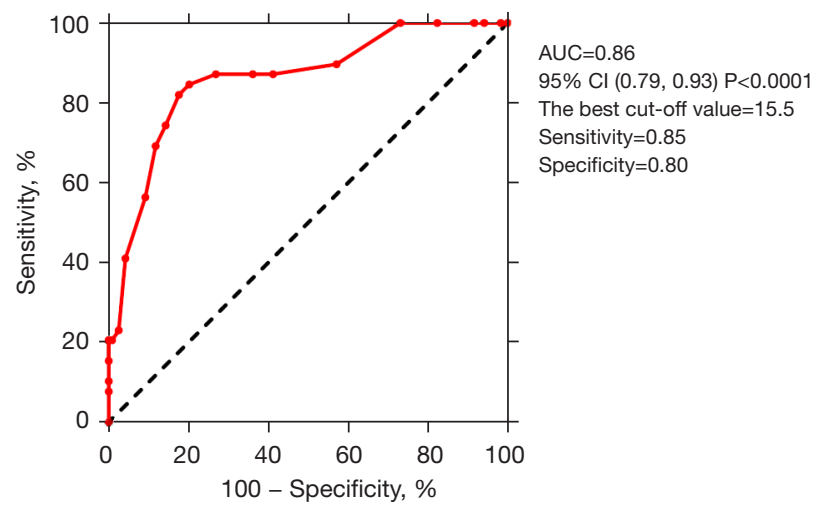

Figure 5 ROC for detection of elevated LAP by BCG-LAP index; LAP was estimated by echocardiography. ROC, receiver operating curve; BCG, ballistocardiography; LAP, left atrial pressure.

Table 5 Accuracy for diagnosis of elevated LAP by BCG-LAP index $(\mathrm{n}=158)$

\begin{tabular}{lcc}
\hline Items & $\begin{array}{c}\text { BCG-LAP index } \\
\text { (cut-off value 15.5) }\end{array}$ & $\begin{array}{c}\text { BCG-LAP index } \\
\text { (cut-off value 18) }\end{array}$ \\
\hline Positive consistent rate & $0.85(0.69,0.94)$ & $0.74(0.52,0.83)$ \\
Negative consistent rate & $0.80(0.71,0.87)$ & $0.86(0.81,0.93)$ \\
Overall agreement & 0.81 & 0.83 \\
PPV & 0.58 & 0.63 \\
NPV & 0.86 & 0.91 \\
\hline
\end{tabular}

BCG-LAP index, parameter related to LAP derived by ballistocardiography; NPV, negative predictive value; PPV, positive predictive value. value of the LAP-index was 15.5 , the positive agreement rate between it and echocardiography LAP was 0.85 , the negative agreement rate was 0.80 , the overall agreement rate was 0.81 , the positive predictive value (PPV) was 0.58 , and the negative predictive value (NPV) was 0.86 . Further, when the cut-off value of the LAP-index was 18 , the positive agreement rate between it and echocardiography LAP was 0.74 , the negative agreement rate was 0.86 , the overall agreement rate was 0.83 , the PPV was 0.63 , and the NPV was 0.91 (Table 5).

\section{Discussion}

\section{Stability and repeatability of the BCG signal acquisition system and LAP calculation algorithm}

The results showed that the output results of THE five different acquisition times were highly consistent. Although subjects 7 and 15 showed varying degrees of body movement in the latter part of the continuous acquisition and recording for 10 minutes, resulting in large variations in the outputs of LAP-index, when observed from the original data, the data during the first 3 minutes of BCG collection was highly stable and reliable. On this basis, prolonging the acquisition time was considered to have a detrimental effect on the stability of the data, so the routine acquisition time for BCG was placed as 1 minute. The test results of the acquisition system between different patients demonstrated that the LAP-index output using the BCG signal acquisition system had reliable repeatability.

\section{Accuracy of the BCG signal acquisition system and LAP calculation algorithm}

Typically, patient with heart failure may be in acute decompensated status or in chronic stable status. Most of HF patient present elevated filling pressure in an acute decompensated status, while in a stable status, the patient's filing pressure may be normal or modestly elevated. The technology here is supposed to detected the significantly elevation of filling pressure, in order to predict an acute decompensated event, before symptom presents. This is the most meaningful clinical value.

We compared results using the LAP-index, a composite index derived from a BCG device with a fiber-optic sensor, with those of echocardiography, which is commonly used in clinical practice. The accuracy to evaluate filing pressure and prognosis performance of comprehensive 
echocardiography is reported to be the best among other non-invasive modalities (22). The results showed that the LAP-index and echocardiography have definite consistency in evaluating LAP, which means that the LAP-index could potentially identify elevated LAP. The LAP-index of the 158 patients ranged from 6 to 32 and compared with the comprehensive evaluation by echocardiography (14), the ROC c-statistic of the index for identifying elevated LAP was 0.86 . Further, when the LAP-index was at the best cutoff value of 15.5, the positive agreement rate between it and echocardiography LAP was 0.85 , the negative agreement rate was 0.80 , the overall agreement rate was 0.81 , the PPV was 0.58 , and the NPV was 0.86 .

To better explore the potential diagnostic capabilities of this non-invasive BCG device we compared the composite LAP-index with the method that is commonly adopted in clinical practice in evaluating LAP and left ventricular filling pressure. A study by Andersen et al. found that (23), compared with the gold standard results of the PCWP, the accuracy of the clinical comprehensive evaluation of pulmonary congestion in patients with heart failure was $72 \%$, the sensitivity was $74 \%$, and the specificity was $69 \%$. In clinical practice, $\mathrm{PCWP} \geq 18 \mathrm{mmHg}$ is usually used as a threshold for evaluating the risk of pulmonary congestion (24). In our study, when the cut-off value of the LAP-index was 18 , the positive agreement rate between it and echocardiography LAP was 0.74 , the negative agreement rate was 0.86 , the overall agreement rate was 0.83 , the PPV was 0.63 , and the NPV was 0.91 . Since this study adopted the comparison standard of the 2016 ASE/ EACVI ultrasound guidelines (14) to comprehensively evaluate LAP, rather than comparison with the gold standard (PCWP or LVDP), the recognition performance of the LAP-index may be affected. Therefore, the conclusions of this study need to be applied cautiously. We also compared the results of our study with other emerging technologies for monitoring pulmonary congestion. Boehmer et al. reported the diagnostic performance of the HeartLogic device (18) (Boston Scientific), which identifies the efficacy of acute heart failure by comparison with NTproBNP, to monitor and manage patients with heart failure. In that study (18), all 76 subjects received HeartLogic and NT-proBNP diagnosis within 48 hours of admission, and the results revealed that the AUC was $0.809(\mathrm{P}<0.0001)$, and when the cut-off value was 1 , the NPV was 0.778 . In our study, when the LAP-index was at the best cut-off value of 15.5 , the positive agreement rate between it and echocardiography LAP was 0.85 , the NPV was 0.86 , the
PPV was 0.58. Although the NPV of the BCG-based LAPindex was higher than the HeartLogic device, it must be noted that the comparison standard used in the HeartLogic study was inconsistent with this study.

In this study, the LAP-index was divided into three intervals according to the E/e' ratio (E/e' $\leq 8,8<\mathrm{E} / \mathrm{e}^{\prime}<15$, $\mathrm{E} / \mathrm{e}^{\prime} \geq 15$ ). The $\mathrm{E} / \mathrm{e}^{\prime} \geq 15$ group's LAP-index was significantly higher than that of the other two groups and there was no statistical difference between the E/e' $\leq 8$ group and the $8<\mathrm{E} / \mathrm{e}^{\prime}<15$ group. These results indicate that the recognition performance of the LAP-index in recognizing a significant increase of LAP is better than that in recognizing a slight increase of LAP. The ultrasound index E/e' is the most feasible and reproducible method for estimating LVFP $(14,25)$. In clinical practice, LAP or PCWP can be estimated by $\left[\mathrm{LAP}=\left(\mathrm{E} / \mathrm{e}^{\prime}\right)+4\right]$ or the Nagueh formula $[\mathrm{LAP}=1.24 \times$ $\left.\left(E / e^{\prime}\right)+1.9\right](23,26)$. While the results of this study revealed that when recognizing $\mathrm{LAP}>18 \mathrm{mmHg}$, the LAP-index had a significant recognition effect, when recognizing LAP in a slight increase $(12-18 \mathrm{mmHg}$ ), there was no obvious difference compared with normal LAP (LAP $<12 \mathrm{mmHg}$ ). These results suggest that a promising use scenario for the LAP-index is in identifying the acute decompensation of heart failure. However, in some clinical situations, E/e' may not be a reliable method for predicting LVFP. Longterm HFrEF patients who have experienced left ventricular remodeling may already have left ventricular dilatation and functional regurgitation of the mitral valve, which leads to an increase in mitral valve inflow, an increase in peak $\mathrm{E}$, and a decrease in the systolic divided by diastolic (S/D) ratio of pulmonary venous blood flow (27). In patients with decompensated heart failure or cardiac resynchronization therapy (CRT), due to obvious mitral regurgitation and abnormal movement of the ventricular septum caused by wide QRS waves, the E/e' has a poor correlation with LVFP. In addition, the tissue Doppler velocity of the mitral valve annulus is affected by heart transplantation or conditions such as mitral valve repair or replacement, severe mitral valve calcification or mitral valve stenosis. Therefore, E/e' is not the best indicator for estimating LAP in subjects with mitral annulus calcification (28). Even in the subgroup with E/e' $<8$, patients who have a certain degree of underlying cardiac disease usually have mild to moderate signs of diastolic weakening. Thus, a comprehensive evaluation parameter such as VTR and LAMVI is required. In our study, to avoid the influence of the above situations, most of the situations which may significantly affect the accuracy of E/e' have been excluded. 
BCG is a non-invasive technique for monitoring cardiovascular parameters which collects chest vibrations caused by the heartbeat and ejection of blood to the peripheral blood vessels (19). BCG technology was reported as early as the end of the $19^{\text {th }}$ century $(19,29)$, and numerous studies were conducted from the 1940s to the 1980s $(30,31)$. However, due to the limitations of computer and sensor technology, it was difficult to detect and analyze BCG waveforms, which limited further study, and after the 1950s, with the rise of other alternative technologies such as echocardiography, BCG technology was gradually abandoned (32). Nowadays, the appearance of smaller, lighter, and more sensitive accelerometers greatly simplifies the measurement and evaluation of BCG signals and opens up new prospects for its clinical applications (19). In 2015, Inan et al. demonstrated that by synchronizing with echocardiography, the waveform of SCG corresponds to valve opening and closing events (19). In 2018, Sørensen et al. reported the vital features of SCG signals, and their relationship with essential demarcation points of the cardiac cycle (33). In the present study, we regarded SCG as a BCG signal measured with an accelerometer, making the SCG signal equivalent to the BCG signal. There have been no reports of a non-invasive product or technology based on BCG for evaluating filling pressures. A possible reason for this is that commonly used BCG/SCG sensor technologies, such as accelerometer chips, may not adequately obtain high-reliability and highrepeatability BCG/SCG signals from the body surface. Our research used a new fiber optic vibration sensor to collect BCG signals. Optical fiber with an outer coating of glass fiber is widely used in communication, and the optical signal is input from one end of the fiber. Under regular circumstances, as a small amount of energy is lost due to the propagation of light in the optical fiber, the optical signal at the output end is almost the same as that at the input end, even when the propagation distance is large. However, if a certain position of the optical fiber creates a bending deformation, the optical signal will be lost at that position and the signal output will be correspondingly weakened. By detecting changes to the optical signal, the bending deformation and changing intensity of the optical fiber can be analyzed (34).

Finally, the core technology of this research is optical fiber sensor based comprehensive algorithm technology, which uses the third heart sound as the major parameter (18). Based on non-invasive hemodynamic information, the comprehensive algorithm (LAP-index) was developed and refined based on the BCG signals from over 1,000 heart disease patients with different ages and underlying diseases in multiple centers. The LAP-index has high reliability and accuracy in identifying elevated LAP, which provides a novel possibility for the non-invasive detection of patients with heart failure. The LAP-index based on fiber optic sensors provides a novel non-invasive option for the remote monitoring of heart failure patients by using hemodynamics instead of monitoring methods, especially for those who need to optimize home management.

\section{Limitations}

To build the algorithm, in the training phase and the testing phase, we separated data in a chronological order rather than a randomized manner. In the training phase, patients with different underlying diseases came from multiple centers in China, and many basic data were missing, while in the testing phase, patients came from a single center, and their basic data were more complete, which may have led to population heterogeneity. However, this heterogeneity is more likely to have reduced the test efficiency of the test set (because it was generated from a different development set), and from this perspective, it may have strengthened the result verification.

In this study, the LAP-index was compared with the 2016 ASE/EACVI ultrasound guidelines for the evaluation of LAP, rather than the gold standard of PCWP or LVEDP. We will verify the composite index and the gold standard in further study. We also believe this device has prospects for use as a medical care device. Combining the diagnosis and clinical evaluation of BCG and natriuretic peptides (NPs), may identify pulmonary congestion in patients with heart failure in emergency situations. Further research is needed to verify this and other potential uses, and to assess its application value in different clinical environments.

\section{Conclusions}

The LAP-index, a composite index derived from BCG and has high reliability and accuracy in identifying elevated LAP. The diagnostic efficacy of the LAP-index in identifying LAP is close to that of the comprehensive evaluation of echocardiography. BCG has the advantages of being non-invasive and easy to operate, which provides a novel possibility for the non-invasive detection of patients with hemodynamic congestion or elevated filling pressure. 


\section{Acknowledgments}

We thank our consultant Professor Carolyn Lam, Senior Consultant from the Department of Cardiology at the National Heart Centre Singapore (NHCS), who provided insight and expertise on cardiac physiology and heart failure, which helped us realize that comparing BCG signals with echocardiography parameters may be a good strategy to understand BCG and ultimately led to the design and conduct of this research. We would also like to extend our gratitude to Professor Jie Cheng, Director of Electrophysiology Basic Research Teaching Staff from the Texas Heart Institute. With his kind help, the engineering team from DARMA Lab and the clinical team from the First Affiliated Hospital of Shantou University Medical College were able to work together on this research.

Funding: This study was funded by the 2019 Shantou Medical Science Talent Cultivation and Clinical Technology Promotion Project (190917105269872).

\section{Footnote}

Reporting Checklist: The authors have completed the STARD reporting checklist. Available at https://dx.doi. org/10.21037/atm-21-5161

Data Sharing Statement: Available at https://dx.doi. org/10.21037/atm-21-5161

Conflicts of Interest: All authors have completed the ICMJE uniform disclosure form (available at https://dx.doi. org/10.21037/atm-21-5161). All authors reported this study was funded by the 2019 Shantou Medical Science Talent Cultivation and Clinical Technology Promotion Project (190917105269872). The payments were made to the First Affiliated Hospital of Shantou University Medical College. Dr. ZC, Dr. QS, Dr. FY, Dr. JH, and Dr. CY reported that they employed by DARMA technology Co., Ltd. (DARMA Lab), China; Dr. SZ reported that he employed by Cardiostory Inc., the United States. The authors have no other conflicts of interest to declare.

Ethical Statement: The authors are accountable for all aspects of the work in ensuring that questions related to the accuracy or integrity of any part of the work are appropriately investigated and resolved. All procedures performed in this study involving human participants were in accordance with the Declaration of Helsinki (as revised in 2013). This research project was approved by the Ethics Review Committee of the First Affiliated Hospital of Shantou University Medical College (No. 2019071). All patients provided written informed consent before participating in this clinical single-center diagnostic trial.

Open Access Statement: This is an Open Access article distributed in accordance with the Creative Commons Attribution-NonCommercial-NoDerivs 4.0 International License (CC BY-NC-ND 4.0), which permits the noncommercial replication and distribution of the article with the strict proviso that no changes or edits are made and the original work is properly cited (including links to both the formal publication through the relevant DOI and the license). See: https://creativecommons.org/licenses/by-nc-nd/4.0/.

\section{References}

1. Taylor CJ, Ryan R, Nichols L, et al. Survival following a diagnosis of heart failure in primary care. Fam Pract 2017;34:161-8.

2. Ponikowski P, Voors AA, Anker SD, et al. 2016 ESC Guidelines for the diagnosis and treatment of acute and chronic heart failure: The Task Force for the diagnosis and treatment of acute and chronic heart failure of the European Society of Cardiology (ESC)Developed with the special contribution of the Heart Failure Association (HFA) of the ESC. Eur Heart J 2016;37:2129-200.

3. Heidenreich PA, Albert NM, Allen LA, et al. Forecasting the impact of heart failure in the United States: a policy statement from the American Heart Association. Circ Heart Fail 2013;6:606-19.

4. Chaudhry SI, Wang Y, Concato J, et al. Patterns of weight change preceding hospitalization for heart failure. Circulation 2007;116:1549-54.

5. Ong MK, Romano PS, Edgington S, et al. Effectiveness of Remote Patient Monitoring After Discharge of Hospitalized Patients With Heart Failure: The Better Effectiveness After Transition -- Heart Failure (BEATHF) Randomized Clinical Trial. JAMA Intern Med 2016;176:310-8.

6. Felker GM, Ahmad T, Anstrom KJ, et al. Rationale and design of the GUIDE-IT study: Guiding Evidence Based Therapy Using Biomarker Intensified Treatment in Heart Failure. JACC Heart Fail 2014;2:457-65.

7. Packer M, Abraham WT, Mehra MR, et al. Utility of impedance cardiography for the identification of shortterm risk of clinical decompensation in stable patients with 
chronic heart failure. J Am Coll Cardiol 2006;47:2245-52.

8. Abraham WT, Compton S, Haas G, et al. Intrathoracic impedance vs daily weight monitoring for predicting worsening heart failure events: results of the Fluid Accumulation Status Trial (FAST). Congest Heart Fail 2011;17:51-5.

9. Lewin J, Ledwidge M, O'Loughlin C, et al. Clinical deterioration in established heart failure: what is the value of BNP and weight gain in aiding diagnosis? Eur J Heart Fail 2005;7:953-7.

10. Abraham WT, Adamson PB, Bourge RC, et al. Wireless pulmonary artery haemodynamic monitoring in chronic heart failure: a randomised controlled trial. Lancet 2011;377:658-66.

11. Conraads VM, Tavazzi L, Santini M, et al. Sensitivity and positive predictive value of implantable intrathoracic impedance monitoring as a predictor of heart failure hospitalizations: the SENSE-HF trial. Eur Heart J 2011;32:2266-73.

12. Yu CM, Wang L, Chau E, et al. Intrathoracic impedance monitoring in patients with heart failure: correlation with fluid status and feasibility of early warning preceding hospitalization. Circulation 2005;112:841-8.

13. van Veldhuisen DJ, Braunschweig F, Conraads V, et al. Intrathoracic impedance monitoring, audible patient alerts, and outcome in patients with heart failure. Circulation 2011;124:1719-26.

14. Nagueh SF, Smiseth OA, Appleton CP, et al. Recommendations for the Evaluation of Left Ventricular Diastolic Function by Echocardiography: An Update from the American Society of Echocardiography and the European Association of Cardiovascular Imaging. J Am Soc Echocardiogr 2016;29:277-314.

15. Capomolla S, Ceresa M, Pinna G, et al. Echo-Doppler and clinical evaluations to define hemodynamic profile in patients with chronic heart failure: accuracy and influence on therapeutic management. Eur J Heart Fail 2005;7:624-30.

16. Brahmbhatt DH, Cowie MR. Remote Management of Heart Failure: An Overview of Telemonitoring Technologies. Card Fail Rev 2019;5:86-92.

17. Thakur PH, An Q, Swanson L, et al. Haemodynamic monitoring of cardiac status using heart sounds from an implanted cardiac device. ESC Heart Fail 2017;4:605-13.

18. Boehmer JP, Hariharan R, Devecchi FG, et al. A Multisensor Algorithm Predicts Heart Failure Events in Patients With Implanted Devices: Results From the MultiSENSE Study. JACC Heart Fail 2017;5:216-25.
19. Inan OT, Migeotte PF, Park KS, et al. Ballistocardiography and seismocardiography: a review of recent advances. IEEE J Biomed Health Inform 2015;19:1414-27.

20. Zhang L, Cai P, Deng Y, et al. Ballistocardiography to identify high left atrial pressure in patients with heart failure. Ann Palliat Med 202 1;10:8155-68.

21. Bozkurt B, Coats AJ, Tsutsui H, et al. Universal Definition and Classification of Heart Failure: A Report of the Heart Failure Society of America, Heart Failure Association of the European Society of Cardiology, Japanese Heart Failure Society and Writing Committee of the Universal Definition of Heart Failure. J Card Fail 2021. [Epub ahead of print].

22. Girerd N, Seronde MF, Coiro S, et al. Integrative Assessment of Congestion in Heart Failure Throughout the Patient Journey. JACC Heart Fail 2018;6:273-85.

23. Andersen OS, Smiseth OA, Dokainish H, et al. Estimating Left Ventricular Filling Pressure by Echocardiography. J Am Coll Cardiol 2017;69:1937-48.

24. Mullens W, Damman K, Harjola VP, et al. The use of diuretics in heart failure with congestion - a position statement from the Heart Failure Association of the European Society of Cardiology. Eur J Heart Fail 2019;21:137-55.

25. Kane GC, Karon BL, Mahoney DW, et al. Progression of left ventricular diastolic dysfunction and risk of heart failure. JAMA 2011;306:856-63.

26. Nagueh SF, Middleton KJ, Kopelen HA, et al. Doppler tissue imaging: a noninvasive technique for evaluation of left ventricular relaxation and estimation of filling pressures. J Am Coll Cardiol 1997;30:1527-33.

27. Taleb M, Khuder S, Tinkel J, et al. The diagnostic accuracy of Doppler echocardiography in assessment of pulmonary artery systolic pressure: a meta-analysis. Echocardiography 2013;30:258-65.

28. Ariza J, Casanova MA, Esteban F, et al. Peak early diastolic mitral annulus velocity by tissue Doppler imaging for the assessment of left ventricular relaxation in subjects with mitral annulus calcification. Eur Heart J Cardiovasc Imaging 2016;17:804-11.

29. Gordon JW. Certain Molar Movements of the Human Body produced by the Circulation of the Blood. J Anat Physiol 1877;11:533-6.

30. Inan OT. Recent advances in cardiovascular monitoring using ballistocardiography. Annu Int Conf IEEE Eng Med Biol Soc 2012;2012:5038-41.

31. MANDELBAUM RA, MANDELBAUM H. The clinical significance of the lateral plane ballistocardiogram. N Y 
State J Med 1957;57:1409-14.

32. Giovangrandi L, Inan OT, Wiard RM, et al.

Ballistocardiography--a method worth revisiting. Annu Int Conf IEEE Eng Med Biol Soc 2011;2011:4279-82.

33. Sørensen K, Schmidt SE, Jensen AS, et al. Definition of Fiducial Points in the Normal Seismocardiogram. Sci Rep
2018;8:15455.

34. Wu T, Liu G, Fu S, et al. Recent Progress of Fiber-Optic Sensors for the Structural Health Monitoring of Civil Infrastructure. Sensors (Basel) 2020;20:4517.

(English Language Editor: B. Draper)

Cite this article as: Zhang L, Cai P, Deng Y, Lin J, Wu M, Xiao Z, Chu Z, Shi Q, Ye F, Hu J, Yang C, Li P, Zhuang S, Wang B. Using a non-invasive multi-sensor device to evaluate left atrial pressure: an estimated filling pressure derived from ballistocardiography. Ann Transl Med 2021;9(20):1587. doi: 10.21037/atm-21-5161 